\title{
Structure of the nucleoli in domestic cattle spermatocytes
}

\author{
Katarzyna Andraszek, Elżbieta Smalec \\ Department of Animal Genetics and Horse Breeding, Institute of Bioengineering \\ and Animal Breeding, Siedlce University of Life Sciences and Humanities, Siedlce, Poland
}

\begin{abstract}
The use of $\mathrm{AgNO}_{3}$ staining, commonly applied in cytogenetics to identify nucleolar organizer regions, has made it possible to identify nucleoli in primary spermatocytes. One nucleolus was identified in each analyzed cell. Considerable morphological differentiation of the nucleoli during the prophase of the first meiotic division, particularly in leptotene, unobserved in other farm animal species, was noticed. Dark-hued grain-like structures were found within the disintegrating nucleoli, corresponding approximately or exactly to the number of the nucleolar organizer regions in the domestic cattle karyotype. Dark areas were identified in the selected prometaphase chromosomes. Their number corresponded with the number of active NORs defined in the domestic cattle karyotype. (Folia Histochemica et Cytobiologica 2012, Vol. 50, No. 3, 346-351)
\end{abstract}

Key words: nucleolus, domestic cattle, bull, spermatogenesis, meiotic chromosomes

\section{Introduction}

The nucleolus is the largest and best explored functional component of the cell nucleus. Ribosomal RNA precursors (pre-rRNA) are synthesized in the nucleolus. It is also instrumental in the generation and maturation of ribosomal RNP molecules (prerRNP). The process is enabled by the fibrillar centres (FC) in which copies of ribosomal gene precursors are transcribed - dense fibrillar components indispensable for the transformation of precursors into target $18 \mathrm{~S}, 5,8 \mathrm{~S}$ and $28 \mathrm{~S}$ rRNA molecules and the granular element that connects with pre-rRNA and rRNA, including the rRNA synthesized outside the nucleolus, which enables the formation of ribonucleoprotein molecules that are precursors of smaller and larger subunits of the mature ribosome. The resultant precursors of ri-

Correspondence address: K. Andraszek, Department of Animal Genetics and Horse Breeding, Institute of Bioengineering and Animal Breeding, Siedlce University of Life Sciences and Humanities, Prusa Str. 14, 08-110 Siedlce, Poland; e-mail: andrasz@uph.edu.pl bosome subunits are transported to the cytoplasm where they mature [1-3].

The nucleolus is a metaphase nuclear structure, perfectly visible with a light microscope. This is due to its considerable density, much greater than the density of the surrounding nucleoplasm and chromatin. The high density stems from a low water content and a high share of dry matter in the nucleolus, ranging from $40 \%$ in large nucleoli to $90 \%$ in small ones [1, 4].

The number of nucleoli in a cell nucleus is determined by the number of active nucleolar organizer regions (NORs). It may be equal to the number of those NOR-chromosomes. Yet, normally, it is lower. This could be due either to the fusion of nucleoli in the interphase nucleus, or the suppression of the activity of certain rDNA loci $[3,5,6]$.

Nucleoli are very dynamic structures. This may be reflected in their cyclical disappearance during mitosis, and reappearance at its end [7]. The nucleolus disappears during cytokinesis and is reproduced in the reconstructed nuclei as a result of NOR activity $[1,4]$. Nucleolar material appears between the chromosomes during the reconstruction of the NOR-associated telophase nucleus. Next, rRNA synthesis is resumed, causing the nucleoli to become more visible. During the interphase, the nucleolus is spherical 
in shape. In the prophase, when the chromosomes become visible, it is evident that the nucleoli are associated with particular nucleolus organising chromosomes $[3,8]$.

Both nucleoli and nucleolar organizer regions have a chemical affinity for heavy metals and can be identified through staining with silver nitrate in a protective colloidal solution of formalin or gelatine [9]. Since nucleolar organizer regions (NORs) determine the structure of nucleoli, an alternative source of information on the activity of rRNA-encoding genes can be found in the analysis of the numbers and sizes of nucleoli in the prophase of the first meiotic division. Throughout the prophase, nucleoli are not degraded. As opposed to mitotic NORs, they are large structures [10-13]. The nucleolus is considered to be one of the strongest promoters of cellular growth, directly associated with the cell cycle and anti-stress reactions. It participates in numerous processes unrelated to ribosome biogenesis, e.g. RNA maturation and control of ontogenesis or telomerase [3, 14-17].

The aim of this study was to determine the number and morphology of nucleoli in the prophase of the first meiotic division in male domestic cattle.

\section{Material and methods}

The analyses were performed on the nucleoli of primary domestic cattle spermatocytes. The experimental materials, testes, were collected post mortem from 20 male domestic cattle aged 1.5 years. According to the presented purpose of the study, the number and shape of the nucleoli of chromosomes in the prophase of the first meiotic division were analyzed. The material for the study was obtained in accordance with the requirements of the National Ethics Committee for Animal Experiments of the European Union (authorization number 37/2011).

The meiotic chromosomes were isolated from the testes immediately after castration using the method described by Evans et al. [18]. Before isolation, the testes were rinsed in $0.88 \%$ sodium citrate. Fragments of the nucleolus (ca. $0.5-1 \mathrm{~cm}^{3}$ ) were macerated in a small volume of $0.88 \%$ sodium citrate, supplemented with fresh $0.88 \%$ citrate up to 10-20 ml, and left for $30 \mathrm{~min}$ at room temperature. After that time, the liquid was transferred from above the sediment into tubes for a 10-minute centrifugation at 1,000 rpm. Subsequently, the supernatant was decanted and the sediment in the tubes was supplemented with fresh sodium citrate up to $5 \mathrm{ml}$. The tubes were then left for $10 \mathrm{~min}$ at room temperature. The resultant suspension was centrifuged again for $10 \mathrm{~min}$ at 1,000 rpm. The supernatant was decanted and the tubes were replenished with fresh Carnoy fixative up to $7 \mathrm{ml}$. The fixing procedure was repeated three times. The fixed cells were suspended in a small amount of fresh Carnoy fixative, spread over degreased slides, and dried at room temperature. The preparations for analysis and the remaining cell suspension were stored at $4^{\circ} \mathrm{C}$.

The preparations were stained according to the method described in 1980 used for staining nucleolar organizer regions of mitotic chromosomes [9]. A $50 \% \mathrm{AgNO}_{3}$ solution and a colloidal gelatine solution were applied on the 1-weekold preparations. The preparations were covered with a coverslip and incubated for $15-20 \mathrm{~min}$ in a thermostat at $60^{\circ} \mathrm{C}$, in complete humidity. After the preparations turned brown in color, the chemical reaction was interrupted and the preparation rinsed several times with distilled water. The preparations were initially examined under a microscope to confirm correct staining. Patchily stained preparations were dyed in a Giemsa solution for $10 \mathrm{~s}$. The preparations were rinsed again in distilled water and dried at room temperature.

The ready preparations were observed using an Olympus BX 50 microscope. The microscopic images were stored in a PC memory using Multiscan software that made it possible to transfer the images from the microscope through the Olympus DP25 digital camcorder connected to the computer. The downloaded images were analyzed using graphic display and statistical programs integrated with the Multiscan system.

For each animal, 50 slides with chromosomes in the first meiotic prophase were analyzed. The microscopic analysis consisted of determining the number of the nucleoli in a cell and their shapes for all the animals in question. The analysis of the nucleolar numbers and shapes employed 1,000 slides with meiotic chromosomes.

\section{Results}

The use of silver nitrate staining enabled the identification of meiotic chromosome nucleoli and the determination of their number and morphology. One nucleolus, well visible both in early and late leptotene cells, was observed in the domestic cattle spermatocytes. Regardless of the preparation quality or staining intensity, all the analyzed preparations were unequivocally found to contain a nucleolus.

The nucleolus becomes gradually degraded throughout the prophase of the first meiotic division. By changing its structure and morphology, it reacts to the changes occurring in the cell nucleus throughout meiosis. The transformations during the prophase of the first meiotic division are the most prominent. At the onset of leptotene in cattle spermatocytes, the nucleoli are regular, almost oval in shape, with a uniform structure (Figure 1A). In the final stage of leptotene, the nucleoli become dispersed (Figure 1B). They still have, however, a compact structure. It is possible to observe small dark structures within the nucleolus whose number is equal to ten or approximately ten. This is the number of the nucleolar organizer regions present in the domestic cattle karyotype. 


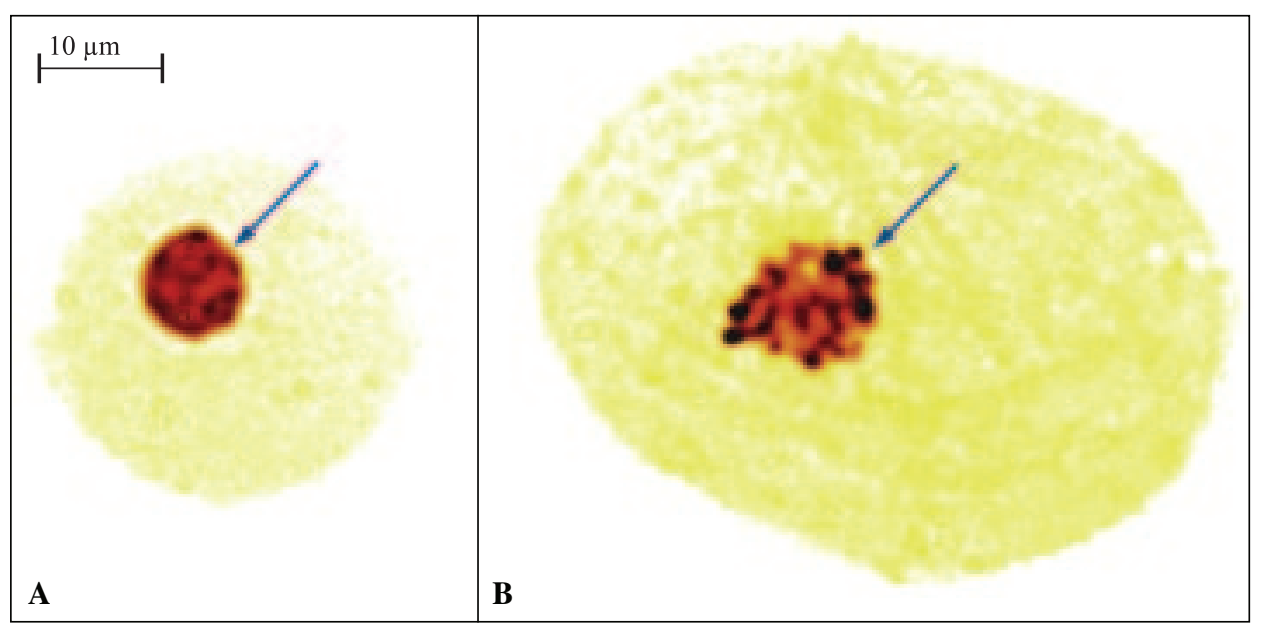

Figure 1. Cells typical of early (A) and late (B) leptotene — the nucleoli are marked with arrows

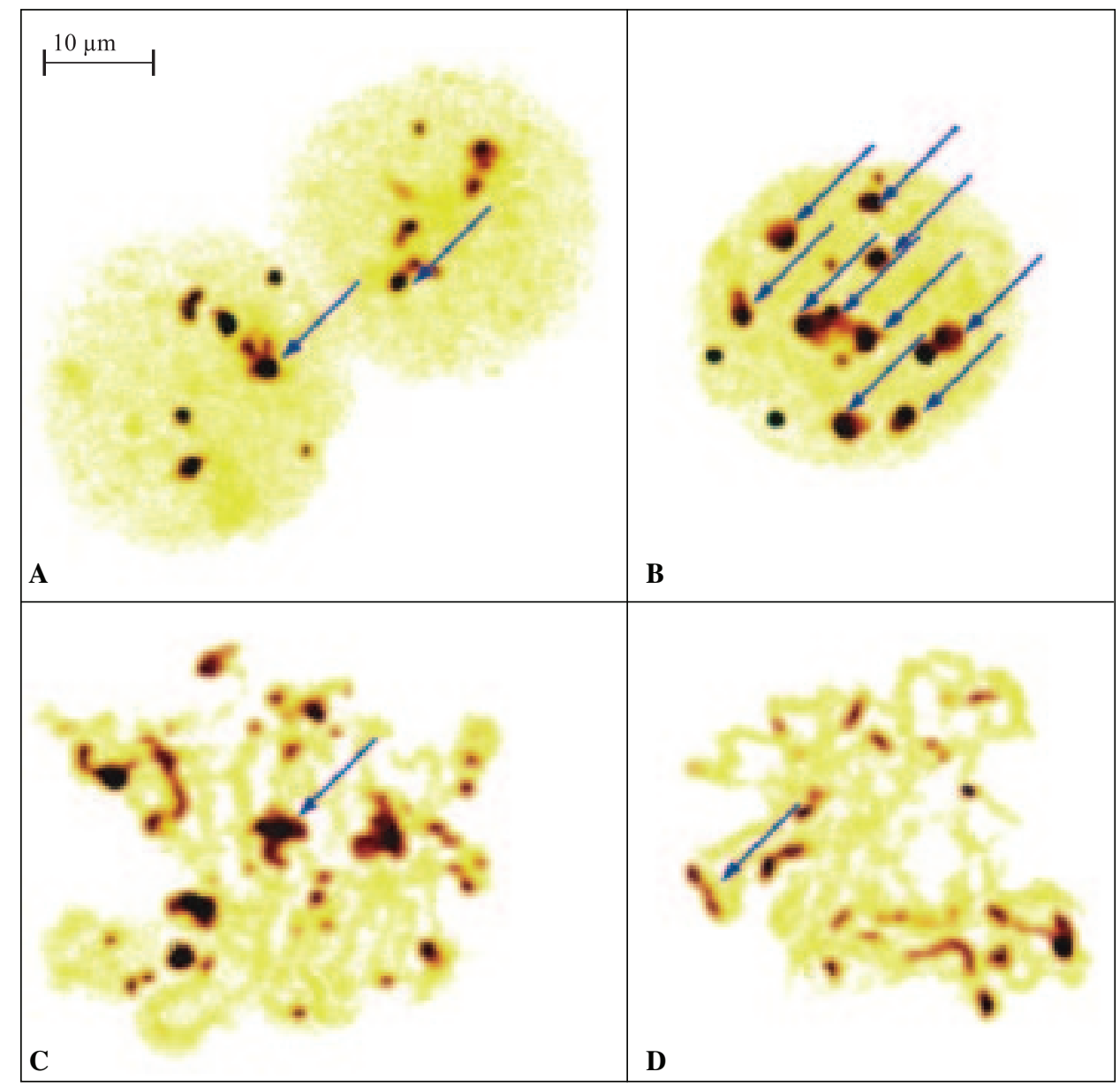

Figure 2. Cells typical of late prophase (A, B) and prometaphase $(\mathbf{C}, \mathbf{D})$. AgNOR staining. The nucleolar organizer regions are marked with arrows

Throughout the prophase, the nucleoli become gradually degraded. However, in the case of domestic cattle spermatocytes, remnants of the nucleoli structures that positively react to silver are observed until the end of the prophase. Their number corresponds with the number of active NORs (Figures 2A, B). In the prometaphase, the nucleolar organizer regions are already clearly associated with particular chromosomes (Figures 2C, D).

Short Giemsa staining of the silver-stained preparations made it possible to even more precisely identify potential nucleolar organizer regions. Potential 


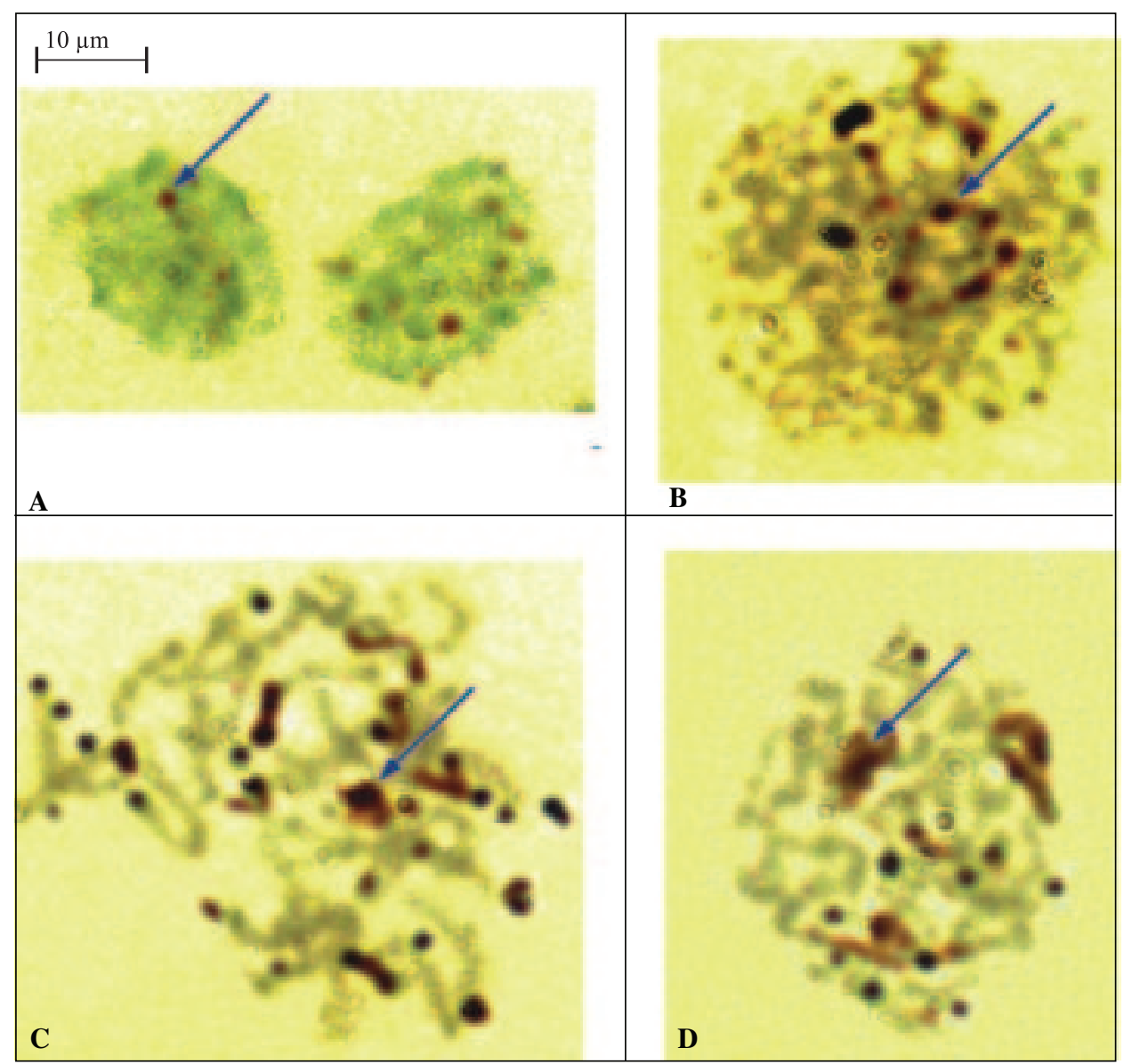

Figure 3. Cells typical of late prophase $(\mathbf{A}, \mathbf{B})$ and prometaphase $(\mathbf{C}, \mathbf{D})$. Ag NOR staining + Giemsa. The nucleolar organizer regions are marked with arrows

NORs were observed as brown points in the background of blue chromatin in the cells typical of late prophase (Figures 3A, B). On the other hand, the nucleolar organizer regions in the prometaphase cells were identified as brown fragments in the selected chromosomes. The blue-hued chromosome chromatin contrasted with the brown NORs (Figures 3C, D).

\section{Discussion}

Domestic cattle are the Bos taurus species within the family Bovidae. The systematized Bovidae karyotype is constituted by 30 autosome pairs and the $\mathrm{X}$ and $\mathrm{Y}$ sex chromosomes. All the autosomes are acrocentric. The sex chromosomes are easy to identify in the karyotype. The $\mathrm{X}$ chromosome is a large submetacentric, while the $\mathrm{Y}$ chromosome is a small metacentric. NORs are present in five autosome pairs $(2,3,4,11$, and 25) in the distal parts of the q arms [19].

A comparison between the results obtained for the number of meiotic nucleoli and the number of active NORs in domestic cattle [19] revealed differences between the number of NORs and nucleoli. Such differences have also been observed in geese [10], goats
[11], hens and quails [12] and horses [13]. These differences are due to the participation of several NORs in the formation of one nucleolus [3]. The lower number of nucleoli compared to that of active NORs results from the fusion of nucleoli in the interphase nucleus and suppression of the activity of some of the rDNA loci [1-3, 12, 13, 20].

The results of the our study show the presence of one nucleolus in a domestic cattle spermatocyte. The available literature contains few analyses of the number and morphology of meiotic nucleoli, or of their connection with the number of active mitotic NORs. Such analyses have been carried out for pigs, with extended attempts to associate the nucleoli with particular bivalents. The cited studies reported, however, different numbers and sizes of cellular nucleoli [21]. On the other hand, domestic goose cells were found to contain between one and four differentlysized nucleoli, the cells predominantly containing one or two nucleoli [10]. Goat spermatocytes have two nucleoli of the same size and different staining intensity [11]. Only one meiotic nucleolus was observed in the domestic horse. Its morphology was correlated with the age of the animal [12]. On the other hand, as 
regards hens and quails, individual animals were observed to have nucleoli in different numbers and sizes. The number of quail nucleoli was one or two. The vast majority of the observed cells contained only one nucleolus. Sporadically, cells with two nucleoli were identified. Domestic hens were also found to have varying numbers of nucleoli. The minimum number of nucleoli in particular cells was one, and the maximum was four. Cells containing two nucleoli were the most common [13].

Different sizes of nucleoli may result from the fact that NORs are differently stained in homologous chromosomes. The experiments confirmed the hypothesis that differentiated cells usually have one large visible silver-stained nucleolus, while non-differentiated cells contain several smaller nucleoli susceptible to silver staining. The size of NORs is a polymorphic characteristic. The polymorphism of nucleolar organizer regions is associated with silver deposits [11, 22]. Otherwise, it is related to differently-sized signals of in situ hybridization [23].

Approximately $12 \%$ of ribosomal DNA blocks do not stain with $\mathrm{AgNO}_{3}$ [24]. These differences account for the presence of differently-sized nucleoli in the prophase of the first meiotic division.

Nucleoli disintegrate throughout the entire prophase of the first meiotic division. The way in which they decay is probably typical of particular vertebrate groups, this being possibly a species-specific characteristic. In the spermatocytes of domestic cattle, the nucleoli gradually become fragmented and 'disintegrate' into tiny structures whose number corresponds with the number of NOR regions. Studies which have analyzed the number and size of nucleoli in avian spermatocytes $[10,13]$ have reported a different mechanism of disappearance of nucleoli. In birds, chromatin reorganization during the prophase of the first meiotic division and the related change in the cell nucleus size is correlated with decreasing sizes of the nucleoli. At the beginning of the prophase, in early leptotene, the nucleoli are visible as large oval structures. At the end of the prophase, in turn, they are observed as tiny points associated with specific bivalents [13].

Nucleolar morphology is also affected by epigenetic mechanisms active at the level of DNA methylation. Entire NORs or only certain nucleolar organizer genes may be suppressed [25].

Apart from its functions being directly connected with ribosome biogenesis, the nucleolus is associated with, or involved in, other cellular processes $[3,5,14$, $26,27]$. Nucleolar morphology has been one of the key criteria of neoplasm classification. The morphometric parameters of nucleoli are the number, size and distance from the nuclear membrane [3, 28-30]. Taking advantage of observations made by Montgomery (1895), biologists dealing with neoplasms quickly tracked down the connection between AgNOR stains and cell proliferation $[2,3,31]$. Most studies during the last few years have exploited the potential offered by the newly discovered nuclear oncogenes connected with the promotion and inhibition of tumors by cytogenetically diagnosed nucleolar mechanisms [3, 32]. The $\mathrm{pRb}$ (the protein of malignant retinoblastoma) and p53 proteins play a major role in the control of the cell cycle progress, as well as ensuring the correct development of daughter cells. These are oncosuppressive proteins, concentrated in the nucleolus [33, 34].

It has also been observed that there is a direct functional link between the nucleolus and p53 control $[4,33]$. Most stress treatments activating p53 also cause the breakdown of the nucleolar structure. A considerable number of somatic cells do not have active telomerase. This limits the number of cell division cycles and suggests that telomerase is a factor in the processes of aging and carcinogenesis $[3,35]$. Telomerase has been found to be present in the nucleolus. Its function is connected with the control nucleolus activity [3]. Along with a class of snoRNA, telomerase locates RNA telomerase within the nucleolus [36].

\section{References}

1. Kłyszejko-Stefanowicz L. Cytobiochemia. PWN, Warszawa 2002;442-468.

2. Derenzini M, Pasquinelli G, O’Donohue MF, Ploton D, Thiry M. Structural and functional organization of ribosomal genes within the mammalian cell nucleolus. J Histochem Cytochem. 2006;54:131-145.

3. Raška I, Shaw PJ, Cmarko D. New Insights into Nucleolar Architecture and Activity. Int Rev Cytol. 2006;255:177-235.

4. Olson MO. The nucleolus. Kluwer Academic Plenum Publisher, London 2004.

5. Gerbi SA, Borovjagin AV, Lange TS. The nucleolus: A site of ribonucleoprotein maturation. Curr Opin Cell Biol. 2003;15:318-325.

6. Hernandez-Verdun D. Nucleolus: From structure to dynamics. Histochem. Cell Biol. 2006;125:127-137.

7. Scheer U, Benavente R. Functional and dynamic aspects of the mammalian nucleolus. BioEssays. 1990;12:14-21.

8. Lam YW, Trinkle-Mulcahy L, Lamond AL. The nucleolus. J Cell Sci. 2005;118:1335-1337.

9. Howell WM, Black DA. Controlled silver-staining of nucleolus organizer regions with a protective colloidal developer a 1-step method. Experientia. 1980;36:1014-1015.

10. Andraszek K, Smalec E. Number and size of nucleoli in the spermatocytes of European domestic goose (Anser anser). Arch Geflügelk. 2007;71:237-240.

11. Andraszek K, Horoszewicz E, Smalec E. Nucleolar organizer regions, satellite associations and nucleoli of goat cells (Capra hircus). Arch Tierz. 2009;52:177-186.

12. Andraszek K, Danielewicz A, Smalec E, Kaproń M. Identification of the nucleoli in domestic horse spermatocytes preliminary investigations. Rocz Nauk PTZ. 2010a;6:13-21. 
13. Andraszek K, Gryzińska M, Knaga S, Wójcik E, Smalec E. Number and size of nucleoli in the spermatocytes of chicken and Japanese quail. Proceedings of 51, Olsztyn, Poland, September 2010. 2010b.

14. Olson MO, Hingorani K, Szebeni A. Conventional and nonconventional roles of the nucleolus. Int Rev Cytol. 2002;219:199-266.

15. Gustafsson MG. Nonlinear structured-illumination microscopy: Wide-field fluorescence imaging with theoretically unlimited resolution. Proc Natl Acad Sci USA. 2005;102:13081$-13086$.

16. Prieto JL, McStay B. Nucleolar biogenesis: the first small steps. Biochem Soc Trans. 2005;33:1441-1443.

17. Hofmann M, Eggeling C, Jakobs S, Hell SW. Breaking the diffraction barrier in fluorescence microscopy at low light intensities by using reversibly photoswitchable proteins. Proc Natl Acad Sci USA. 2005;102:17565-17569.

18. Evans EP, Breckon G, Ford CE. An airdrying method for meiotic preparation from mammalian testes. Cytogenetics. 1964;15:289-294.

19. Di Berardino D, Di Meo GP, Gallagher DS, Hayes H, Iannuzzi L. International System for Chromosome Nomenclature of Domestic Bovids (ISCNDB 2000). Cytogenet Cell Genet. 2001;92:283-299.

20. Raška I, Koberna K, Malinsk J, Fodlerova H, Masata M. The nucleolus and transcription of ribosomal genes. Biol Cell. 2004;96:579-594.

21. Słota E. Chromosome polymorphism in swine (in Polish). Rocz. Nauk. Zoot. Instytut Zootechniki Kraków, Dissertation. 1998;7:1-58.

22. Mellink CHM, Bosma AA, De Hann NA. Variation in size of Ag-NORs and fluorescent rDNA in situ hybridization signals in six breeds of domestic pig. Hereditas. 1994;120: 141-149.

23. Babu V, Rao Ghosh K, Mohanty D. Nucleolar organising region evaluation using new NOR FISH probe. Indian J Hum Genet. 2005;11:44-46.
24. Kurihara Y, Suh DS, Suzuki H, Moriwaki K. Chromosomal locations of Ag-NORs and clusters of ribosomal DNA in laboratory strains of mice. Mamm Genome. 1994;5:225-228.

25. Grummt I, Pikaard CS. Epigenetic silencing of RNA polymerase I transcription. Nat Rev Mol Cell Biol. 2003;4:641-649.

26. Pederson T. The plurifunctional nucleolus. Nucleic Acids Res. 1998;26:3871-3876.

27. Santoro R, Grummt I. Molecular mechanisms mediating methylation dependent silencing of ribosomal gene transcription. Mol Cell. 2001;8:719-725.

28. Nafe R, Schlote W. Histomorphometry of brain tumours. Neuropathol Appl Neurobiol. 2004;30:315-328

29. Smetana K, Klamova H, Pluskalova M, Stockbauer P, Jiraskova I, Hrkal Z. Intranucleolar translocation of AgNORs in early granulocytic precursors in chronic myeloid leukaemia and K 562 cells. Fol Biol (Praha). 2005;51:89-92.

30. Smetana K, Klamova H, Pluskalova M, Stockbauer P, Hrkal Z. To the intranucleolar translocation of AgNORs in leukemic early granulocytic and plasmacytic precursors. Histochem Cell Biol. 2006;125:165-170.

31. Derenzini M, Pession A, Trere D. Quantity of nucleolar silver-stained proteins is related to proliferating activity in cancer cells. Lab Invest. 1990;63:137-140.

32. Oskarsson T, Trumpp A. The Myc trilogy: Lord of RNA polymerases. Nat Cell Biol. 2005;7:215-217.

33. Ryan KM, Philips AC, Vousden KH. Regulation and function of the p53 tumor suppressor protein. Curr Opin Cell Biol. 2001;13:332-337.

34. Trere D, Ceccarelli C, Montanaro L, Tosti E, Derenzini M. Nucleolar size and activity are related to pRb and p53 status in human breast cancer.JHistochem Cytochem. 2004; 52:1601-1607.

35. Maser RS, DePinho RA. Keeping telomerase in its place. Nat Med. 2002;8:934-936.

36. Lukowiak AA, Narayanan A, Li ZH, Terns RM, Terns MP. The snoRNA domain of vertebrate telomerase RNA functions to localize the RNA within the nucleus. RNA. 2001;7:1833-1844. 